\title{
An abundant biliary metabolite derived from dietary omega-3 polyunsaturated fatty acids regulates triglycerides
}

\author{
Trisha J. Grevengoed, ${ }^{1}$ Samuel A.J. Trammell, ${ }^{1}$ Jens S. Svenningsen, ${ }^{1}$ Mikhail V. Makarov, ${ }^{2}$ Thomas Svava Nielsen, \\ Jens Christian Brings Jacobsen, ${ }^{3}$ Jonas T. Treebak, ${ }^{1}$ Philip C. Calder, ${ }^{4,5}$ Marie E. Migaud, ${ }^{2}$ Benjamin F. Cravatt, ${ }^{6}$ and \\ Matthew P. Gillum ${ }^{1}$
}

\begin{abstract}
1Novo Nordisk Foundation Center for Basic Metabolic Research, Faculty of Health and Medical Sciences, University of Copenhagen, Copenhagen, Denmark. ${ }^{2}$ Mitchell Cancer Institute, Department of Pharmacology, University of South Alabama, Mobile, Alabama, USA. ${ }^{3}$ Department of Biomedical Sciences, Faculty of Health and Medical Sciences, University of Copenhagen, Copenhagen, Denmark. ${ }^{4}$ Faculty of Medicine, University of Southampton, Southampton, United Kingdom. ${ }^{5}$ National Institute for Health Research (NIHR) Southampton Biomedical Research Centre, University Hospital Southampton NHS Foundation Trust and University of Southampton, Southampton, United Kingdom. ${ }^{\circ}$ Departments of Cell Biology and Chemistry, The Scripps Research Institute, La Jolla, California, USA.
\end{abstract}

\begin{abstract}
Omega-3 fatty acids from fish oil reduce triglyceride levels in mammals, yet the mechanisms underlying this effect have not been fully clarified, despite the clinical use of omega-3 ethyl esters to treat severe hypertriglyceridemia and reduce cardiovascular disease risk in humans. Here, we identified in bile a class of hypotriglyceridemic omega-3 fatty acid-derived $\mathrm{N}$-acyl taurines (NATs) that, after dietary omega-3 fatty acid supplementation, increased to concentrations similar to those of steroidal bile acids. The biliary docosahexaenoic acid-containing (DHA-containing) NAT C22:6 NAT was increased in human and mouse plasma after dietary omega-3 fatty acid supplementation and potently inhibited intestinal triacylglycerol hydrolysis and lipid absorption. Supporting this observation, genetic elevation of endogenous NAT levels in mice impaired lipid absorption, whereas selective augmentation of C22:6 NAT levels protected against hypertriglyceridemia and fatty liver. When administered pharmacologically, C22:6 NAT accumulated in bile and reduced high-fat diet-induced, but not sucroseinduced, hepatic lipid accumulation in mice, suggesting that C22:6 NAT is a negative feedback mediator that limits excess intestinal lipid absorption. Thus, biliary omega-3 NATs may contribute to the hypotriglyceridemic mechanism of action of fish oil and could influence the design of more potent omega-3 fatty acid-based therapeutics.
\end{abstract}

\section{Introduction}

Omega-3 fatty acids are essential to many cellular functions, but are not made by mammals and instead are found in highest abundance in fatty fish and fish oil supplements (1). When consumed in pharmacologic doses, omega-3 fatty acids and their synthetic derivatives markedly reduce circulating triacylglycerol (TAG) levels and liver TAG in humans $(2,3)$ and are effective in treating severe hypertriglyceridemia (4). The essential omega-3 fatty acids eicosapentaenoic acid (EPA, C20:5) and docosahexaenoic acid (DHA, C22:6) reduce TAG and inflammation in part by decreasing lipid synthesis, increasing fatty acid oxidation, inhibiting adipocyte lipolysis, and displacing the omega- 6 fatty acid arachidonic acid (C20:4) from phospholipids, thereby preventing proinflammatory mediator synthesis (1). An EPA derivative, ethyl eicosapentaenoic acid, also protects against ischemic events in high-risk patients with elevated triglycerides (5), while a liver-targeted analog of

\section{Related Commentary: https://doi.org/10.1172/JCl147558}

Conflict of interest: The authors have declared that no conflict of interest exists. Copyright: ( 2021, American Society for Clinical Investigation.

Submitted: September 2, 2020; Accepted: January 20, 2021; Published: March 15, 2021. Reference information: J Clin Invest. 2021;131(6):e143861.

https://doi.org/10.1172/JCl143861.
EPA, icosabutate, improves circulating and hepatic lipid profiles, reduces inflammation and fibrosis, and is currently being studied in human trials to treat nonalcoholic steatohepatitis (NASH) (6). NASH is a common consequence of chronic nonalcoholic fatty liver disease (NAFLD), a malady present in approximately $25 \%$ of the global population that is expected to increase further alongside the metabolic syndrome (7). Nevertheless, despite their therapeutic utility and expanding clinical applications, how omega-3 fatty acids and their derivatives improve triglyceride homeostasis has not been fully elucidated. A better understanding of these mechanisms of action could point the way to increasingly potent and effective omega-3-based therapeutics.

We hypothesized that $N$-acyl taurines (NATs), amphipathic lipids composed of a variable fatty acyl chain and taurine, might contribute to the triglyceride-lowering effects of omega-3 fatty acids in vivo. We postulated this because, even though the proportion of omega-3 fatty acids in hepatic lipids is low, a DHA-derived NAT (C22:6 NAT) is the most abundant NAT species in liver at baseline and after pharmacological inhibition of NAT degradation $(8,9)$. The unusual enrichment of omega-3 fatty acyl chains in NATs and their high turnover in liver suggest an undefined biological role potentially related to polyunsaturated fatty acid (PUFA) homeostasis.

NATs are found in multiple tissues, undergo rapid clearance in the liver, and are hydrolyzed by the enzyme fatty acid amide hydro- 
lase (FAAH) (10). FAAH degrades several classes of acyl amides beyond NATs, including $N$-acyl ethanolamines (NAEs), which include several biologically active molecules (11-13). A conventional loss-of-function model for FAAH causes the accumulation of both NAEs and NATs, making it impossible to disentangle the function of these classes of molecules in this model. Previously, we reported the generation and evaluation of a mouse model carrying a point mutation in FAAH (FAAH-S268D). FAAH-S268D mice degrade NAEs normally but are unable to hydrolyze NATs, causing NATs to accumulate (9), thus allowing us to characterize the function of NATs in vivo. The conventional FAAH-knockout mouse, with high levels of NATs and NAEs, rapidly develops fatty liver and becomes insulin resistant $(14,15)$. In contrast, FAAH-S268D mice with elevated endogenous NATs alone display improved insulin sensitivity and increased glucagon-like peptide 1 (GLP-1 secretion. Similarly, acute treatment with C18:1 NAT increases the secretion of glucoregulatory hormones, improves glucose tolerance, and decreases appetite, leading to reduced food intake (9).

In mouse liver, the NAT lipid family is enriched in polyunsaturated acyl-chains, and these species increase the most when FAAH activity is limited $(8,9)$, despite the very low abundance of omega-3 fatty acids relative to other fatty acids. These observations led us to explore the physiology of PUFA-containing NATs in vivo by increasing substrate availability through omega-3 fatty acidrich fish oil feeding as well as exogenous treatment with specific NATs. We found that the DHA-containing NAT, C22:6 NAT, greatly increases with omega-3 fatty acid supplementation, especially in bile. Of note, in the 1960s, medium-chain NATs were found to be the primary emulsifier in crayfish digestive juice (16), suggesting an important role in digestion and intestinal function. However, the presence and function of NATs in mammalian bile have not been previously explored. We hypothesized that C22:6 NAT would mediate some proportion of the beneficial effects of omega- 3 fatty acid supplementation, including lowering hepatic and plasma TAG levels. Congruent with this idea, C22:6 NAT limited lipid absorption in both WT and FAAH-S268D mice and prevented high-fat diet-mediated (HFD-mediated) hepatic lipid accumulation in FAAH-S268D mice. The action of C22:6 NAT in FAAH-S268D mice, which cannot degrade NATs normally, indicates that this intact molecule, rather than its DHA and taurine components, confers beneficial effects on fatty liver and hypertriglyceridemia.

\section{Results}

NATs are enriched in bile, and omega-3 fatty acid supplementation increases C22:6 NAT amounts. We previously showed that NATs containing polyunsaturated acyl chains are abundant in liver and plasma and greatly increase with impaired NAT hydrolysis and that these species could be metabolites that mediate positive phenotypes, such as high GLP-1 secretion (9). Standard rodent chow contains very little EPA or DHA, so substrate could limit the amount of NATs made from these PUFAs. To overcome this limit, $\mathrm{C} 57 \mathrm{Bl} / 6 \mathrm{NTac}$ mice were fed a HFD (45\% kcal fat) containing primarily lard (HFD) or $20 \% \mathrm{kcal}$ from menhaden fish oil (approximately $5 \% \mathrm{kcal}$ from EPA/DHA). Because NATs are taurine-conjugated amphipathic lipids, similar to steroidal bile acids, we examined the metabolome of bile to check for the presence of NATs (Figure 1A). Interestingly, we found that NATs were highly concentrated in bile relative to plasma, regardless of diet (Figure $1, \mathrm{~B}$ and $\mathrm{C}$ ), reaching concentrations in the range of steroidal bile acids. In fish oil-fed mice, primarily PUFA-containing NATs were elevated in both bile and plasma, indicating a strong substrate preference (Figure 1, B and C). We detected species containing unusual acyl chains, such as C22:7 NAT, but structure, such as branching of the acyl chain, was not determined because of a lack of standards and difficulty in synthesis. Humans that consumed EPA/DHA supplements (13.1 g/week for 12 months, ref. 17) had plasma C20:5 NAT and C22:6 NAT levels that were elevated over baseline without altering the C18:1 NAT concentration (Figure 1D). These data show that the amount of C22:6 NAT greatly increases with DHA supplementation in both mice and humans, indicating potential substrate preference in synthesis, and that C22:6 NAT is concentrated in the bile, potentially for disposal to control hepatic DHA concentrations or to regulate intestinal function.

C22:6 NAT lowers lipid absorption through inhibition of TAG degradation. To understand whether increasing NATs in bile affects dietary lipid absorption, C57Bl/6NTac mice were fed a HFD or a fish oil diet for 2 weeks and then subjected to an oil absorption test. Fish oil feeding slowed the appearance of TAG after oil gavage (Figure 2A), but did not change the secretion of endogenous TAG, as indicated by VLDL levels (Figure 2B). Because biliary NATs were greatly altered by fish oil feeding, we tested whether a specific NAT could be responsible for slowing lipid absorption. Mice were given an oil gavage containing a $\left.{ }^{3} \mathrm{H}\right]$ triolein tracer, with or without purified C18:1 NAT, C20:4 NAT, or C22:6 NAT. Interestingly, of the tested NATs, only C22:6 NAT decreased plasma radioactivity 2 hours after oil gavage (Figure 2, B and C). C22:6 NAT also lowered the amount of radiolabel found in intestinal cells, indicating that the lipid had not been able to be taken up into enterocytes (Figure $2 \mathrm{E}$ ). To determine whether the deficiency was due to impaired fatty acid uptake into enterocytes or to low TAG hydrolysis, mice were given an oil gavage containing a $\left[{ }^{14} \mathrm{C}\right]$ oleic acid tracer with or without C22:6 NAT. We found that C22:6 NAT did not impair the appearance of plasma or intestinal fatty acids (Figure 2, F and G), indicating that intestinal uptake of lipids was not impaired. Unlike the weight-loss drug orlistat (18), C22:6 NAT did not directly impair pancreatic lipase activity (Figure 2H). C22:6 NAT did reduce TAG hydrolysis in a lipid emulsion (Figure 2I), and when added to bovine bile, C22:6 NAT reduced the size of micelles (Figure 2J), which can inhibit lipase activation (19). Taken together, these data indicate that the specific increase of C22:6 NAT in the intestine decreased lipid absorption by impairing TAG hydrolysis.

Genetic ablation of NAT hydrolysis impairs lipid absorption. FAAH-S268D mice cannot hydrolyze NATs and display elevated plasma and hepatic NAT levels (9). Here, we detected elevated NATs in bile from FAAH-S268D mice, particularly PUFA-containing NATs (Figure 3A), similar to what we observed in fish oil-fed WT mice (Figure 1A). We also observed impaired lipid absorption with normal VLDL secretion in the FAAH-S268D mice (Figure 3, $\mathrm{B}$ and $\mathrm{C}$ ), and fecal acylglycerol, including TAG and monoacylglyerol, was elevated in FAAH-S268D mice after 4 weeks on a very HFD (60\% fat diet, Figure 3D). The impairment in absorption was specific to lipids, as maltodextrin was digested and absorbed as glucose at normal rates (Figure 3E). Further, intestinal permeability and upper gut transit speed were not altered (Figure 3, F and 
A

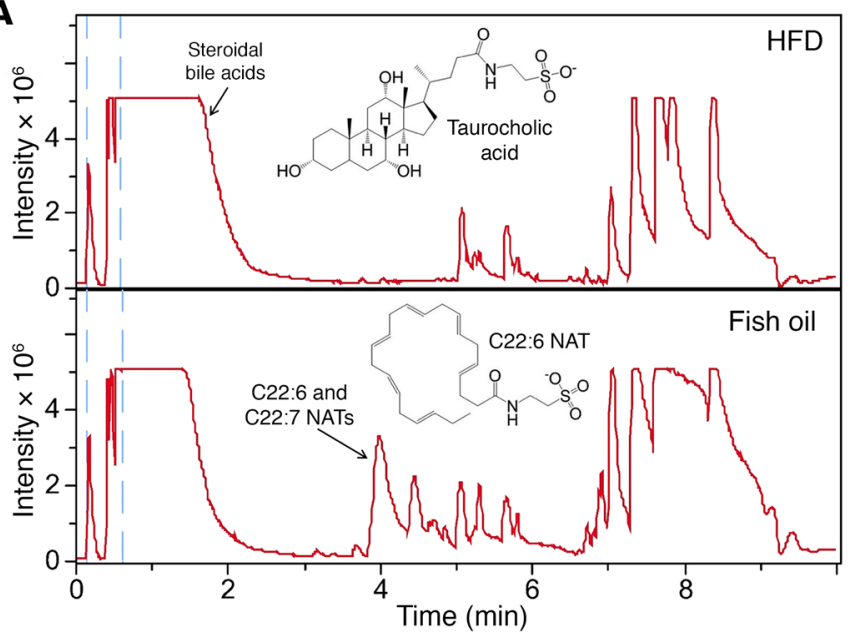

B

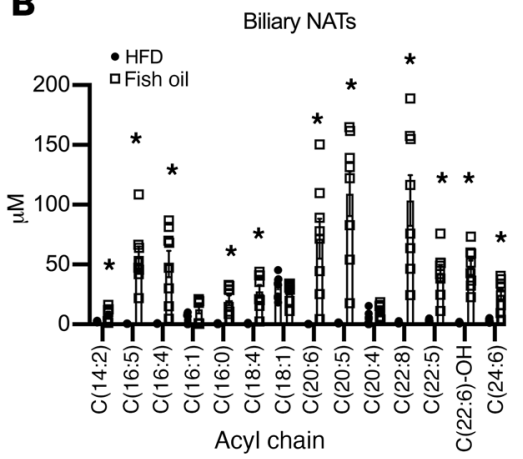

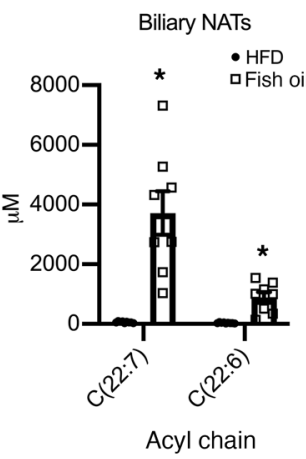

C

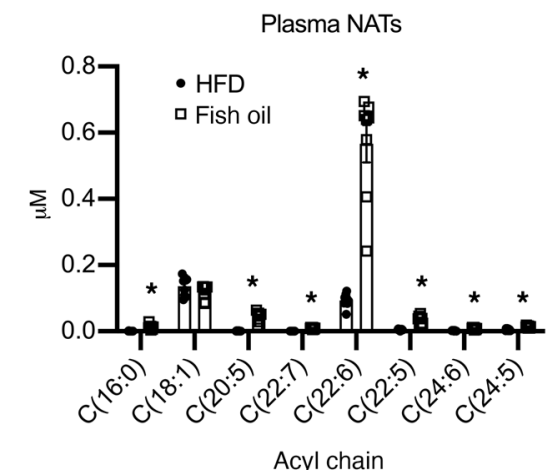

Acyl chain
D Human plasma
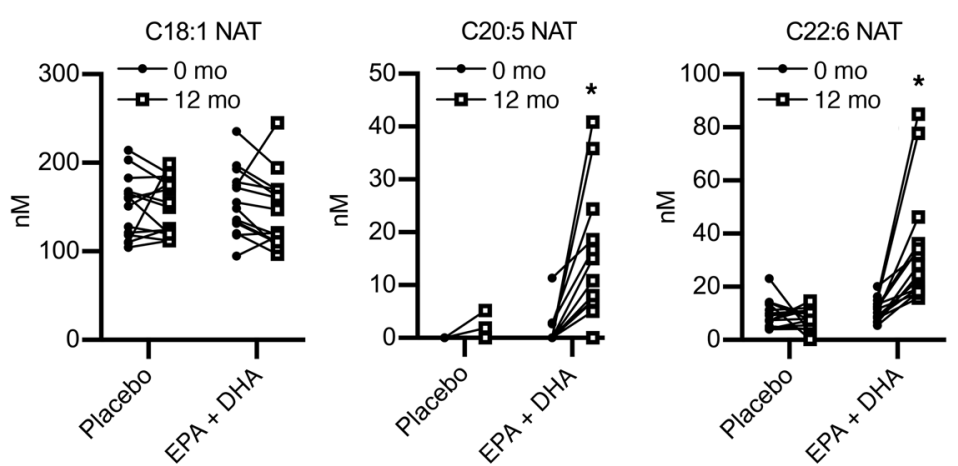

Figure 1. NATs are enriched in bile, and omega-3 fatty acid supplementation increases C22:6 NAT amounts. (A) Representative chromatogram of murine bile after 3 days of a lard-based HFD or a fish oil diet. The indicated peaks denote steroidal bile acids or C22:6 and C22:7 NATs. Insets show the structures of the taurine-conjugated biliary lipids taurocholate and C22:6-NAT. Biliary (B) and plasma (C) NATs after 3 days of HFD or fish oil feeding ( $n=8-10)$. (D) Human plasma after EPA/DHA supplementation (13.1 g/week) or placebo oil $(n=14)$. Data are presented as the mean \pm SEM. ${ }^{*} P<0.05$ between treatments by FDR-corrected $t$ test (A-C) or 2-way ANOVA (D).

G). The lipid absorption defect in FAAH-S268D mice was probably not due to altered steroidal bile acids, as bile secretion was higher (Figure 3H), and steroidal bile acid content (Figure 3I) and bile acid synthesis gene expression were normal (Figure 3J). Thus, the change in composition and quantity of NATs in bile was a likely cause of altered lipid absorption in the FAAH-S268D mice.

Impaired NAT hydrolysis alters whole-body energetics. Despite a deficiency in lipid absorption, chow-fed FAAH-S268D mice had normal body weight (BW) and composition at 10-12 weeks of age (Figure 4A). Food intake and physical activity were also not altered in the FAAH-S268D mice compared with FAAH-WT mice (Figure 4, B and C). Interestingly, oxygen consumption $\left(\mathrm{VO}_{2}\right)$ and carbon dioxide output $\left(\mathrm{VCO}_{2}\right)$ were low, leading to low energy expenditure values (Figure $4, D-G$ ). This low energy expenditure may compensate for a calorie deficit due to low lipid absorption.

Fish oil feeding of FAAH-S268D mice prevents hypertriglyceridemia and hepatic lipid accumulation. FAAH-S268D mice were next fed a moderately HFD ( $45 \%$ kcal fat) or a matched fish oil diet to determine whether omega-3 fatty acid supplementation could further increase C22:6 NAT and increase its effects. We found that fish oil feeding significantly increased many species of NATs
(Supplemental Figure 1; supplemental material available online with this article; https://doi.org/10.1172/JCI143861DS1), with large increases in biliary and plasma C22:6 NAT (Figure 5, A and B). Fish oil-fed FAAH-WT mice had levels of plasma and biliary C22:6 NAT similar to those in HFD-fed FAAH-S268D mice, which cannot hydrolyze NATs. Interestingly, the defect in lipid absorption was less pronounced in HFD-fed FAAH-S268D mice (Figure 5C) than in chow-fed mice (Figure 3B), indicating that some compensation to normalize lipid absorption is possible on a moderate-fat diet. However, fish oil-fed FAAH-S268D mice had lower plasma TAG levels after an oil meal than did FAAH-WT mice, without altering VLDL secretion (Figure 5, C and D), indicating an impairment in lipid absorption.

Despite impaired lipid absorption, FAAH-S268D mice gained weight normally on both the HFD and fish oil diet (Figure 5E). Food intake was not different in the first 3 days of diet feeding (Supplemental Figure 1). In both genotypes, fish oil feeding caused the expected effects, such as lower fat mass (Figure 5, F and G) and plasma cholesterol levels (Figure 5H). Interestingly, with fish oil feeding, FAAH-S268D mice had more lean mass than did mice in the other groups (Figure 5F), and FAAH-S268D mice had low plas- 
A
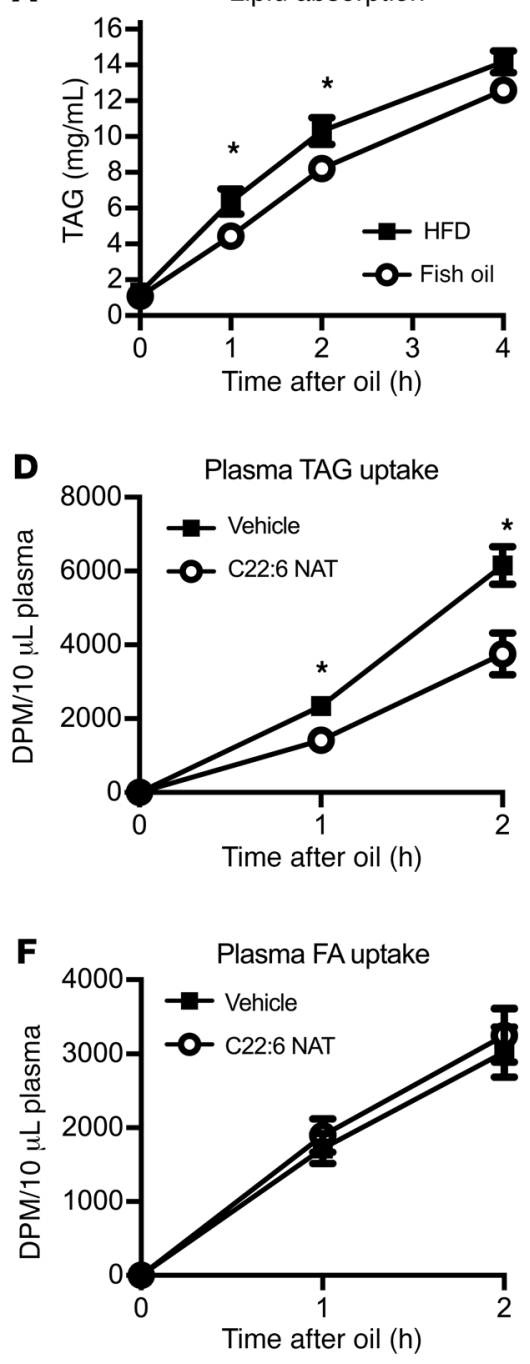

B

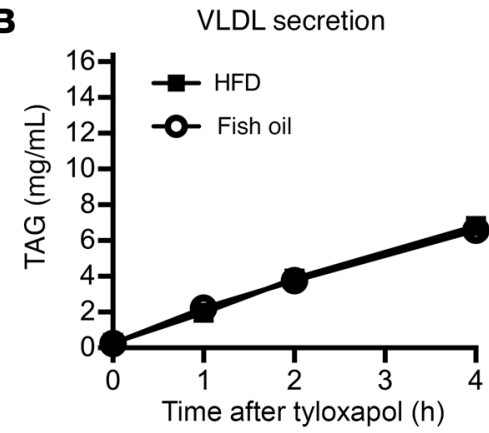

E

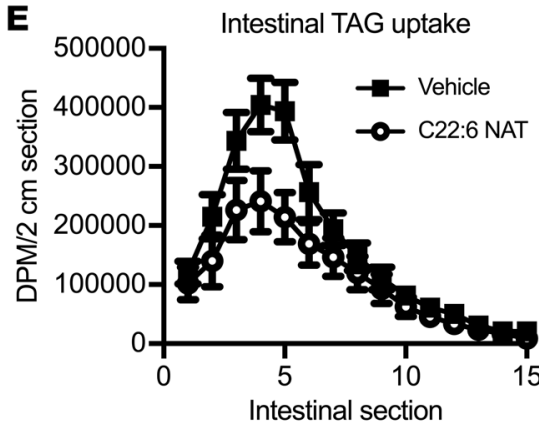

G

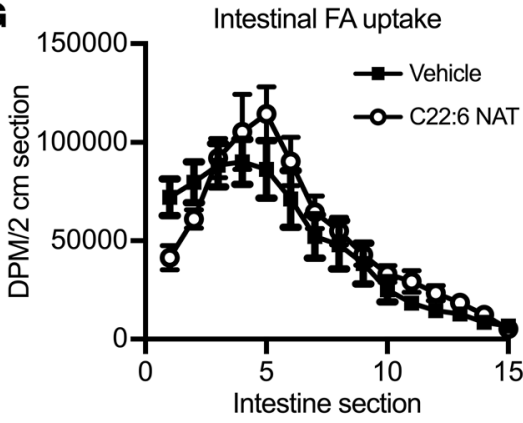

C Plasma TAG uptake
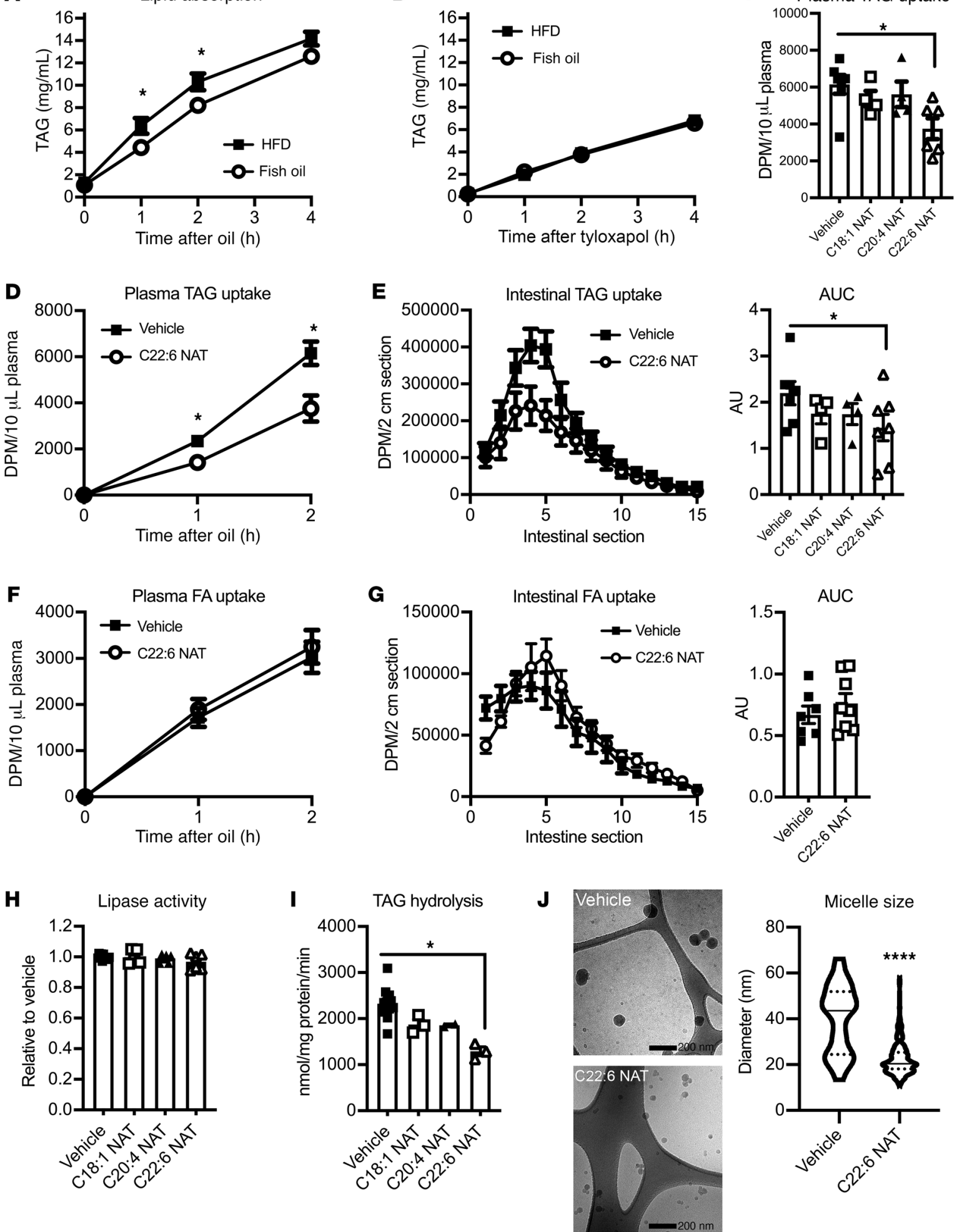

Figure 2. C22:6 NAT lowers lipid absorption by inhibiting TAG degradation. (A and B) Plasma TAG with gavage of olive oil (lipid absorption; $n=11-12$ ) or after inhibition of lipoprotein lipase alone (VLDL secretion; $n=4$ ). (C-E) ${ }^{3} \mathrm{H}$ in plasma or small intestine after lipoprotein lipase inhibition and oral gavage of oil with $\left[{ }^{3} \mathrm{H}\right]$ triolein $\pm 100 \mu \mathrm{M}$ NAT $(n=4-7)$. (F and $\mathbf{~ C ) ~}{ }^{14} \mathrm{C}$ in plasma or small intestine after lipoprotein lipase inhibition and oral gavage of oil with $\left[{ }^{14} \mathrm{C}\right]$ oleic acid $\pm 100 \mu \mathrm{M}$ C22:6 NAT ( $n=7-8$ ). (H) Lipase activity measured using 4-nitrophenyl palmitate $\pm 100 \mu \mathrm{M}$ NAT $(n=2$ separate experiments in triplicate). (I) Lipase-mediated TAG hydrolysis measured using $\left[{ }^{3} \mathrm{H}\right]$ triolein in a taurocholate emulsion $\pm 100 \mu \mathrm{M}$ NAT ( $n=3$ separate experiments in triplicate). (J) Representative images and quantification of size of bovine bile micelles $\pm 500 \mu \mathrm{M} \mathrm{C22:6} \mathrm{NAT} \mathrm{imaged} \mathrm{by} \mathrm{cryo-EM.} \mathrm{Micelles} \mathrm{are} \mathrm{the} \mathrm{small,} \mathrm{dark} \mathrm{circles.} n=96$ (vehicle); $n=772$ (C22:6 NAT). Scale bars: $200 \mathrm{~nm}$. Data are presented as the mean \pm SEM. ${ }^{*} P<0.05$ between treatments using $t$ tests (A, B, D, F, and G) or 1-way ANOVA (C, E, H, and I) and ${ }^{* * *} P<0.001$ between conditions using a Mann-Whitney $U$ test (J). FA, fatty acid. 
A

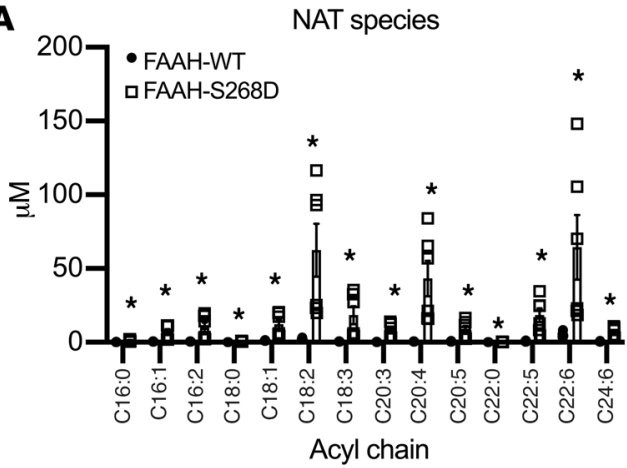

B

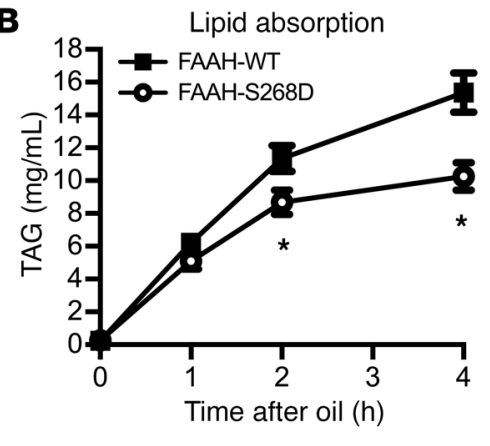

F Intestinal permeability

D Fecal acylglycerol

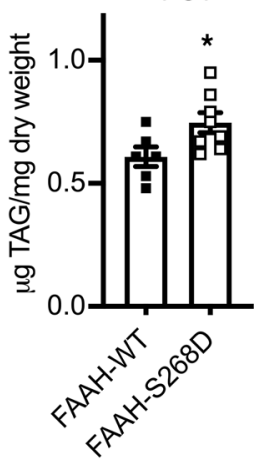

E

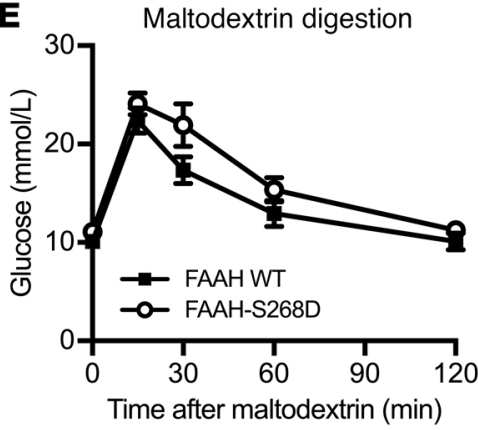

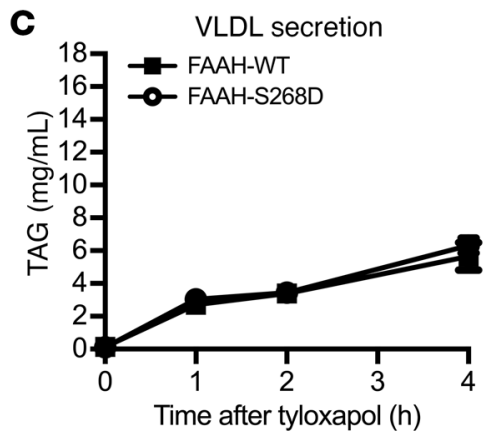

G

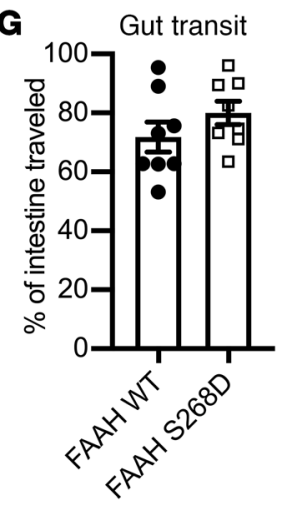

H

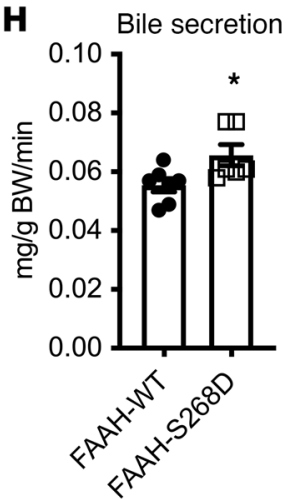

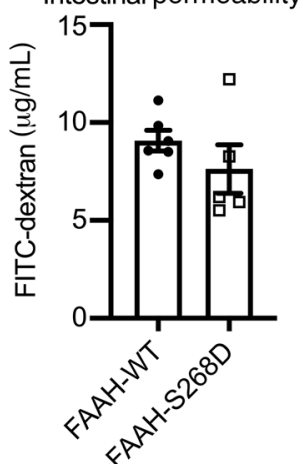

I

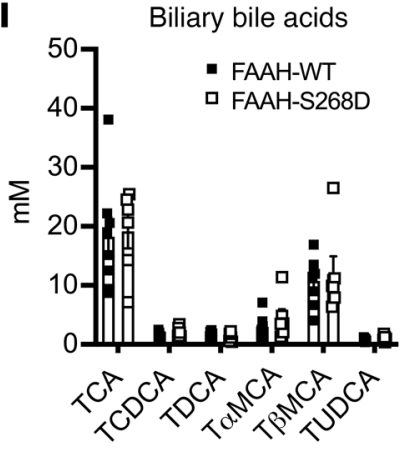

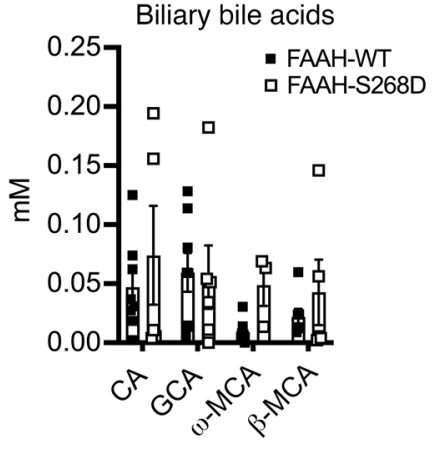

J

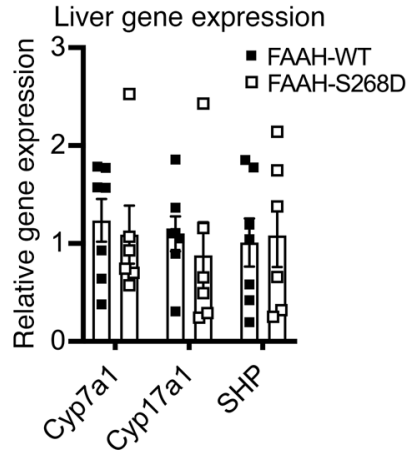

Figure 3. Genetic ablation of NAT hydrolysis impairs lipid absorption. (A) Biliary NAT in FAAH-WT and FAAH-S268D mice ( $n=6-8)$. (B and C) Plasma TAC after gavage of sunflower oil (lipid absorption; $n=7-8$ ) or after inhibition of lipoprotein lipase alone (VLDL secretion; $n=7$ ). (D) Fecal acylglycerol after 4 weeks of very HFD feeding ( $60 \% \mathrm{kcal}$ from fat; $n=6-8)$. (E) Oral maltodextrin digestion $(n=8)$. (F) Plasma FITC-dextran 4 hours after oral gavage $(n=5-6)$. (C) Upper gut transit measured 30 minutes after gavage of a mixed meal containing $10 \%$ charcoal $(n=8)$. (H) Hepatic bile secretion rate $(n=6-8)$. (I) Biliary steroidal bile acids $(n=6-8)$. TCA, taurocholic acid; TCDCA, taurochenodeoxycholic acid; TDCA, taurodeoxycholic acid; T $\alpha$ MCA, tauro- $\alpha$-muricholic acid; TaMCA, tauro- $\beta$-muricholic acid; TUDCA, tauroursodeoxycholic acid; CA, cholic acid; GCA, glycocholic acid; $\omega$-MCA, $\omega$-muricholic acid; $\beta$-MCA, $\beta$-muricholic acid. (J) Hepatic gene expression $(n=5-7)$. Data are presented as the mean \pm SEM. ${ }^{*} P<0.05$ compared with FAAH-WT using $t$ tests.

ma TAG levels, regardless of diet (Figure 5I). Nonesterified fatty acid (NEFA) levels were unchanged (Figure 5J). Fish oil feeding decreased liver size (Figure $5 \mathrm{~K}$ ) and TAG levels in both genotypes, but FAAH-S268D livers were more substantially protected, as they contained less than half the TAG levels measured in FAAHWT livers (Figure 5L). We found no differences between the genotypes in terms of hepatic expression of inflammatory markers or genes that regulate lipid metabolism, possibly indicating a mechanism other than altered fatty acid oxidation or synthesis (Figure $5 \mathrm{M})$. Taken together, these data indicate that highly increasing PUFA-containing NATs allows mice to gain weight normally while being protected from developing fatty liver, potentially because of impaired dietary lipid absorption. The fact that the greatest effects occurred in mice unable to break down NATs demonstrates that NATs themselves may be the beneficial molecules.

C22:6 NAT treatment of FAAH-S268D mice mimics the effects of fish oil feeding. Because C22:6 NAT was the only tested NAT able to impair lipid absorption (Figure 2, C and D) and was highly elevated with fish oil feeding, we tested whether treatment of FAAHS268D mice with exogenous C22:6 NAT could have an effect similar to that of fish oil feeding. After s.c. injection, C22:6 NAT peaked in plasma after 30 minutes (Figure 6A), and the C22:6 
A

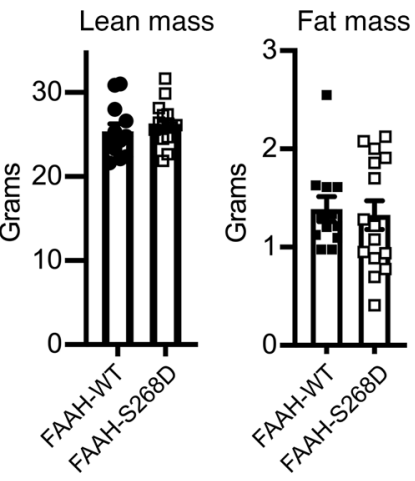

E

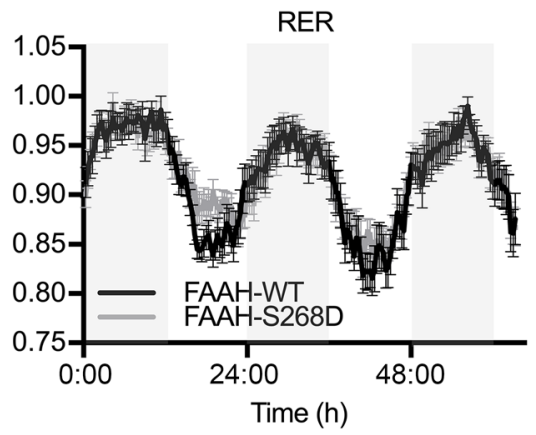

G

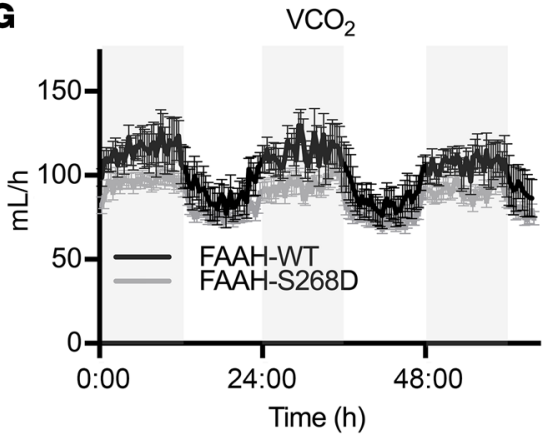

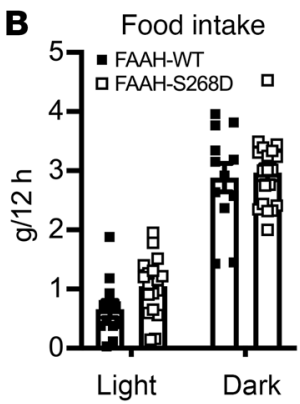

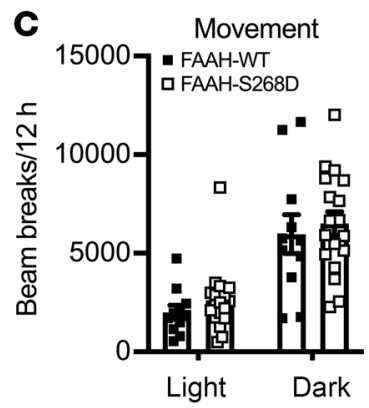

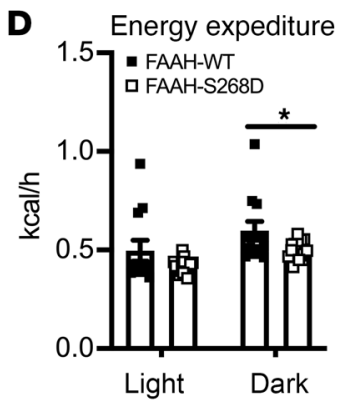

$\mathbf{F}$
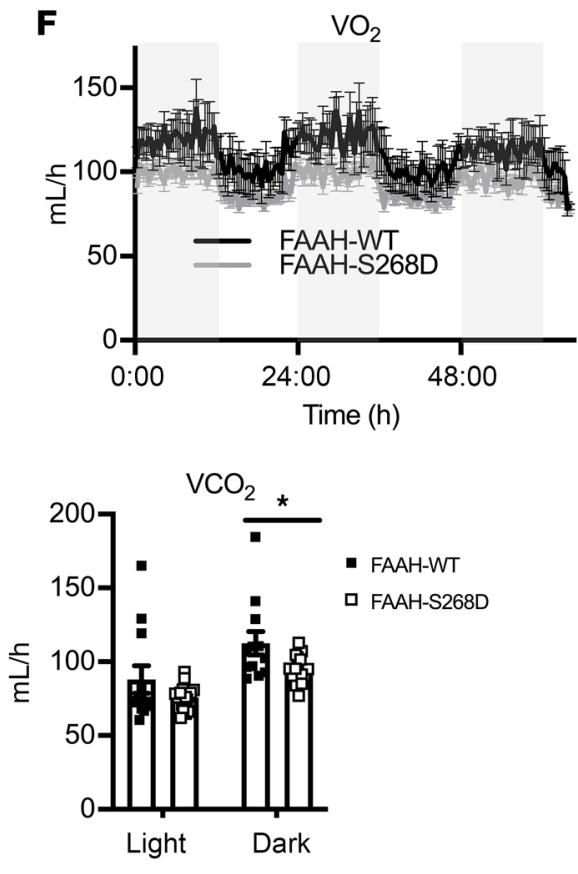

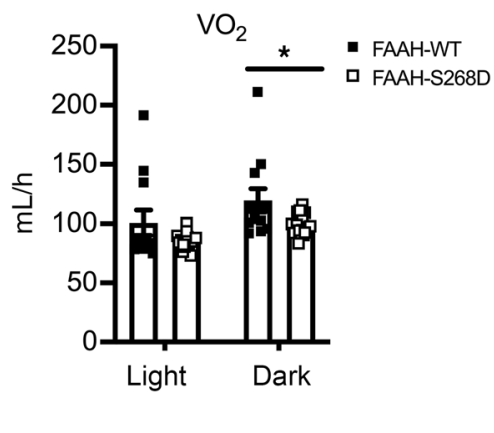

Figure 4. Impaired NAT hydrolysis alters whole-body energetics. (A) Body composition in chow-fed, 10- to 12-week-old male mice, measured by MRI ( $n=12-15)$. (B-G) Metabolic chambers measured food intake, physical activity, heat output, respiratory exchange ratio (RER), VO ${ }_{2}$, and VCO, over at least 72 hours $(n=6-10)$. Data are presented as the mean \pm SEM. ${ }^{*} P<0.05$ compared with FAAH-WT by $t$ test.

NAT concentration in bile rose until the last tested point of 2 hours (Figure 6B), indicating that when FAAH-mediated NAT hydrolysis is impaired, NATs can be cleared from plasma into bile. When FAAH-S268D mice were given a lipid absorption test 2 hours after C22:6 NAT injection, TAG appearance was diminished 2 hours and 4 hours after oil gavage, indicating that lipid absorption was impaired (Figure 6C), similar to what was observed with dosing of C22:6 NAT directly with the meal (Figure 2C).

In a study of chronic treatment, FAAH-S268D mice were fed a HFD (45\% kcal fat) and received a daily s.c. injection of vehicle (PBS) or C22:6 NAT (10 mg/kg) for 2 weeks. C22:6 NAT treatment did not significantly affect weight gain or inguinal adipose weight, but it decreased gonadal adipose weight (Figure 6, D-F). Consistent with fish oil feeding in FAAH-S268D mice, C22:6 NAT treatment lowered plasma TAG and glucose levels and tended to lower plasma cholesterol (Figure 6, G-I). We observed no treatment differences with NEFAs (Figure 6J) or ketones (Figure 6K). C22:6 NAT-treated mice had 37\% less hepatic TAG (Figure 6L), indicating that C22:6 NAT is partly responsible for the effect of fish oil in a model of delayed NAT hydrolysis. No signs of fibrosis, inflammation, or necrosis were seen with either treatment (Figure 6M). C22:6 NAT had no observed effect in WT mice (data not shown), probably due to rapid hydrolysis and limited excretion in bile. Overall, in a model of prolonged NAT circulation and biliary content, C22:6 NAT treatment was able to diminish HFD-induced fatty liver development and improve several blood parameters without altering weight gain.

A high-sucrose diet prevents C22:6 NAT-mediated protection against fatty liver. To this point, we have shown that C22:6 NAT caused lipid malabsorption and opposed HFD-induced fatty liver. We reasoned that the lipid malabsorption is responsible for decreased hepatic lipid deposition; however, we have not ruled out that C22:6 NAT may directly affect lipid metabolism in the liver. To determine whether C22:6 NAT-mediated lipid malabsorption is necessary to prevent fatty liver, FAAH-S268D mice were fed a high-sucrose diet (HSD) to induce fatty liver from car- 
A

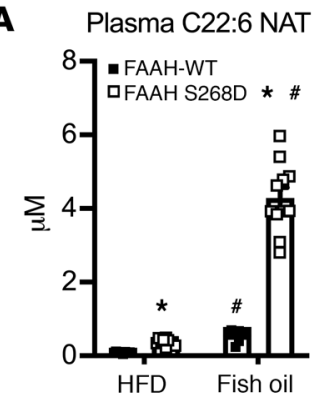

E

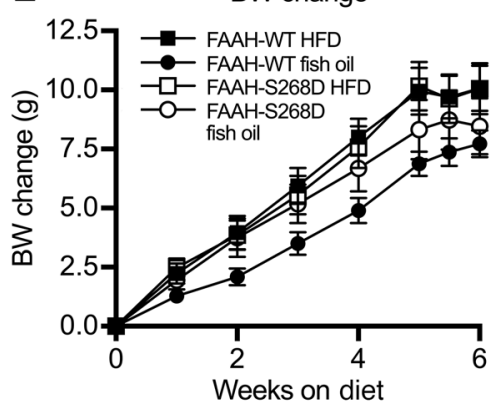

Bile C22:6 NAT

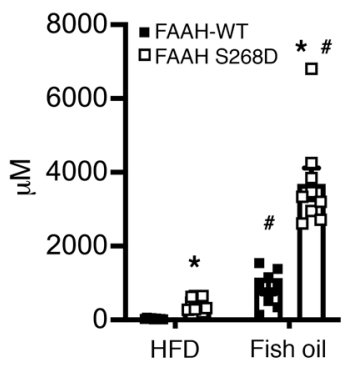

$\mathbf{F}$

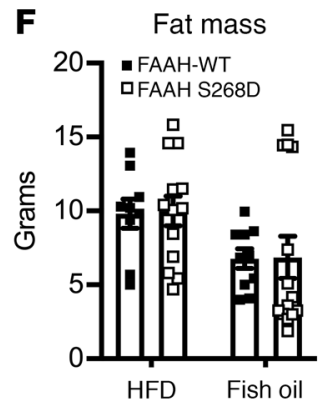

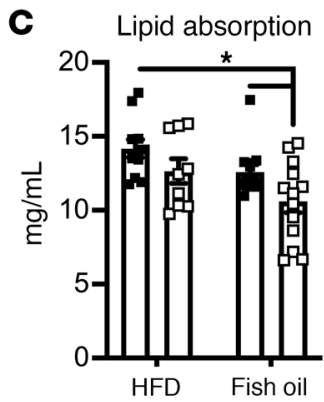

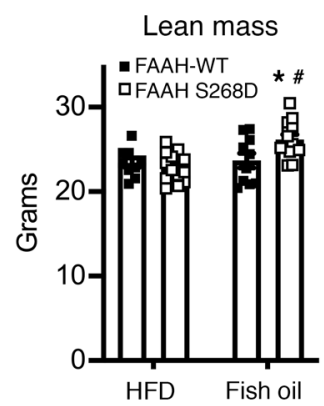

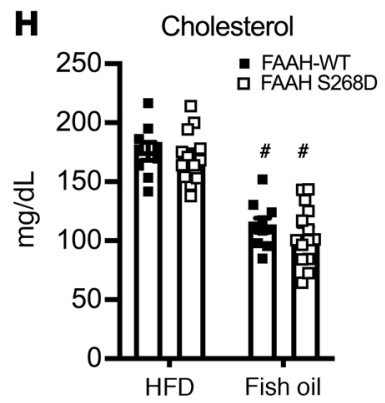

$\mathbf{L}$

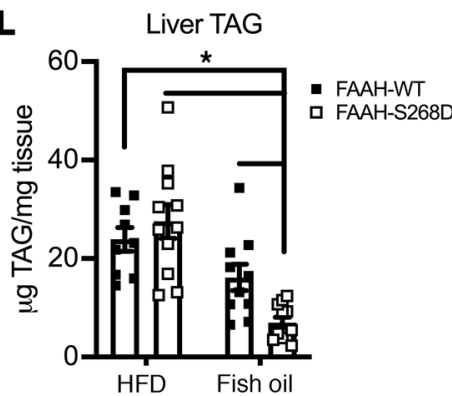

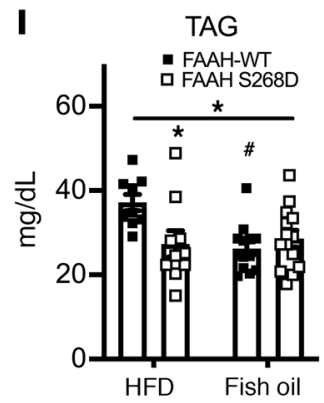

J

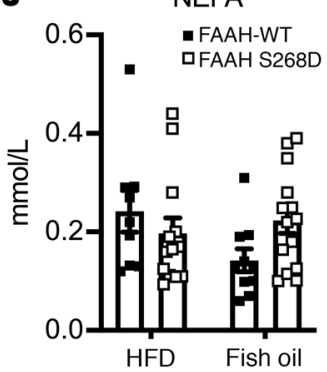

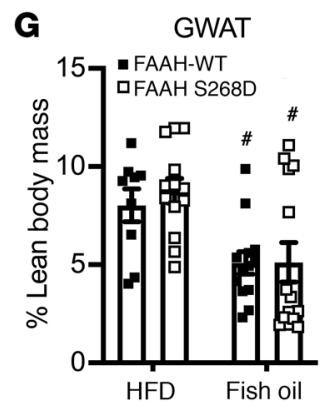
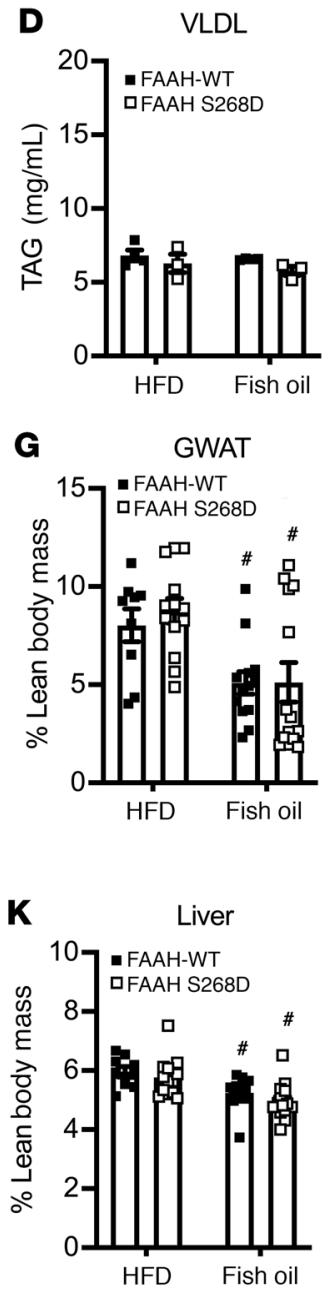

M

Hepatic gene expression

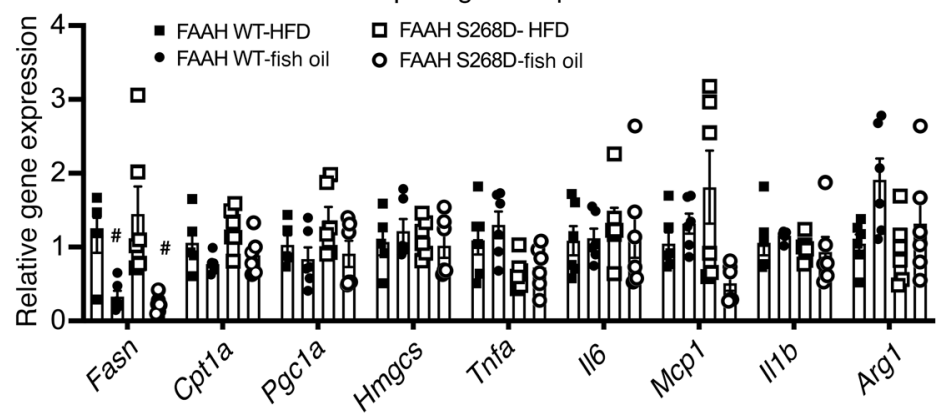

Figure 5. Fish oil feeding of FAAH-S268D mice prevents hypertriglyceridemia and hepatic lipid accumulation. Plasma (A) and biliary (B) C22:6 NAT after 3 days of a lard-based HFD (45\% kcal fat) or a matched diet containing $20 \% \mathrm{kcal}$ from fish oil ( $n=8-11)$. (C and D) Plasma TAC with gavage of olive oil (lipid absorption; $n=9-13$ ) or after inhibition of lipoprotein lipase alone (VLDL secretion; $n=3-4)$ ). (E) BW change over the 6-week diet period ( $n=9-14)$. (F) Total fat and lean mass after 5 weeks of the indicated diets $(n=9-14)$. (G) Gonadal white adipose tissue (GWAT) weight $(n=9-14)$. (H-J) Plasma lipid levels ( $n=$ 9-14). (K) Liver weight ( $n=9-14)$. (L) Liver TAG levels after 6 weeks on the indicated diets $(n=9-14)$. (M) Hepatic expression of lipid metabolism genes and markers of inflammation $(n=6)$. Data are presented as the mean \pm SEM. ${ }^{*} P<0.05$ compared with FAAH-WT mice, by 2 -way ANOVA; ${ }^{\#} P<0.05$ compared with HFD feeding within the genotype, by 2-way ANOVA.

bohydrate consumption instead of dietary lipid absorption. With a HSD, we observed that BW gain, gonadal adipose weight, food intake, and plasma measures were unaltered by C22:6 NAT treatment (Figure 7, A-D). Importantly, HSD-fed FAAH-S268D mice treated with C22:6 NAT were no longer protected from liver TAG accumulation (Figure 7E), indicating that dietary lipid is key to C22:6 NAT protection.

GLP-1 levels increase in models of impaired lipid absorption $(20,21)$ and can have beneficial effects on metabolism, including diminishing fatty liver $(22,23)$. We tested whether C22:6 NAT 
A

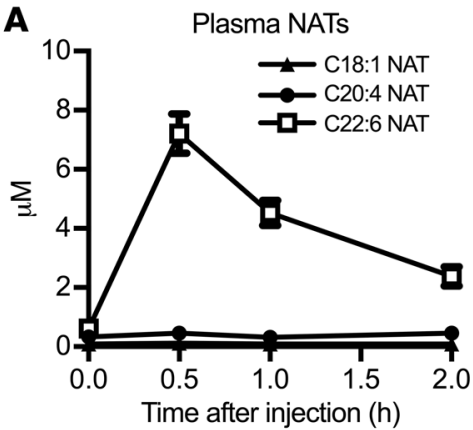

B

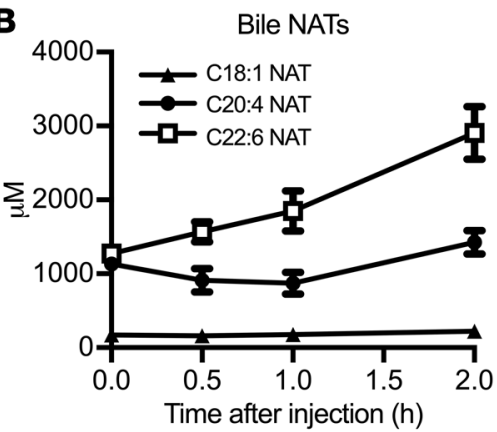

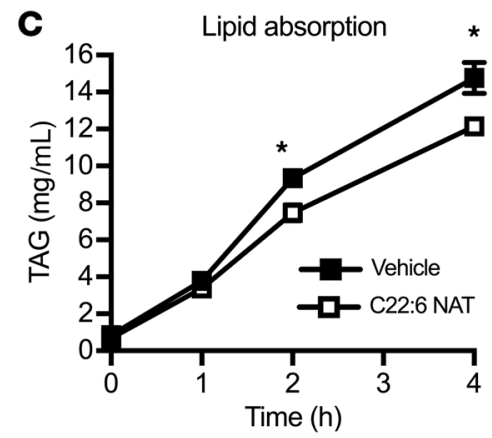

D

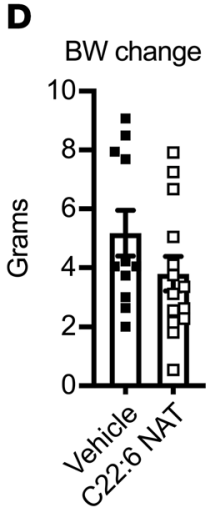

E

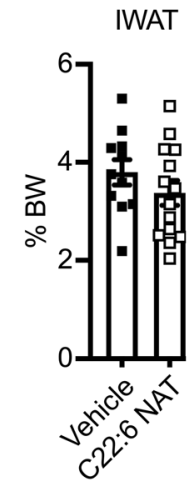

K Acetoacetate

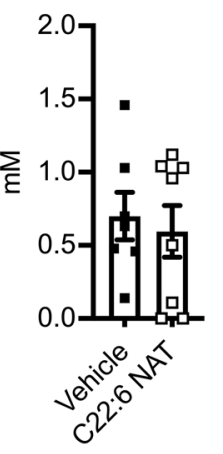

$\beta$-hydroxybutyrate

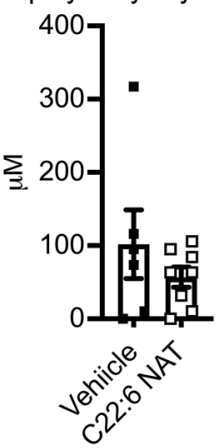

$\mathbf{F}$

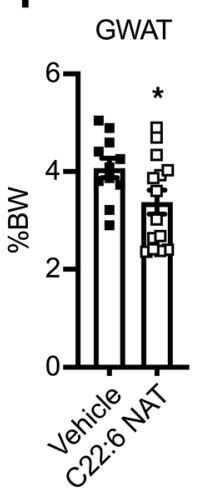

G

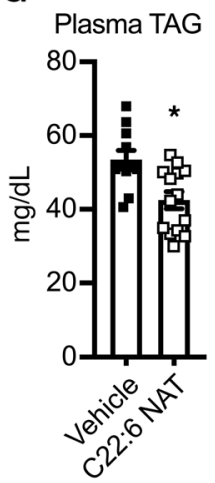

H Plasma cholesterol

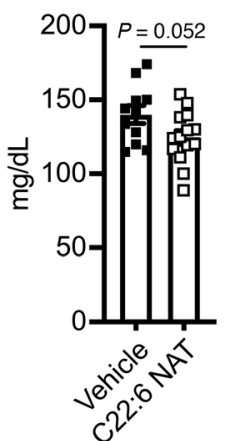

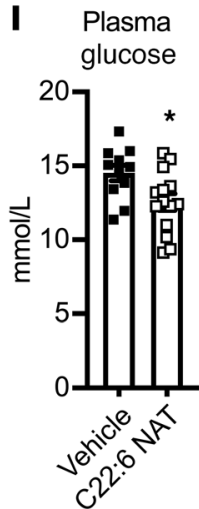

J Plasma

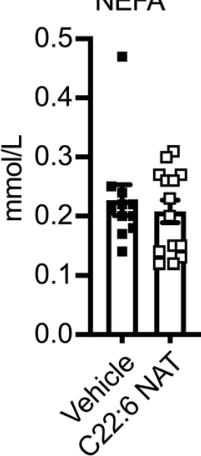

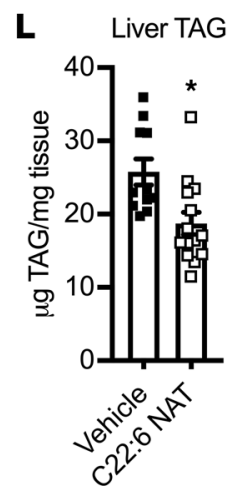
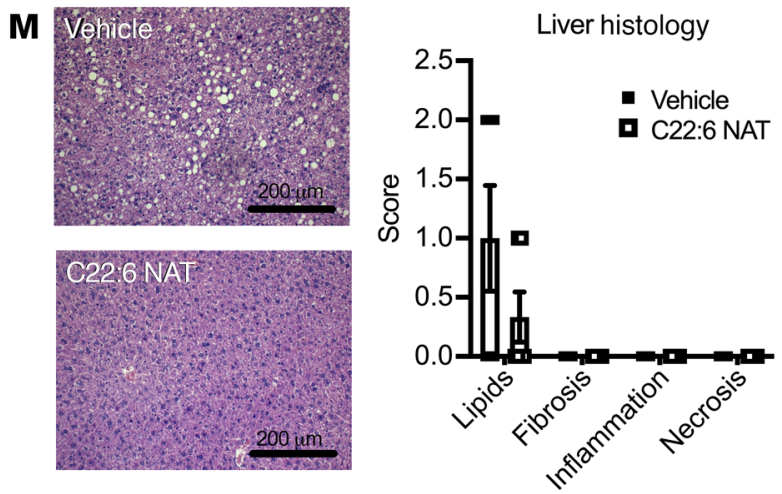

Figure 6. C22:6 NAT treatment of FAAH-S268D mice mimics the effects of fish oil feeding. C22:6 NAT (10 mg/kg) was administered s.C. to FAAH-S268D mice, and plasma (A) and bile (B) were analyzed for changes in NATs $(n=3-5)$. (C) Plasma TAG levels with gavage of olive oil and lipoprotein lipase inhibition 2 hours after s.c. injection of C22:6 NAT (10 mg/kg) or vehicle (PBS) ( $n=9-10)$ into FAAH-S268D mice. (D-M) FAAH-S268D mice were fed a lard-based HFD (45\% kcal fat) for 2 weeks and given daily s.c. C22:6 NAT (10 mg/kg) or vehicle (PBS) ( $n=11-16)$. (D) BW change over a 2-week period. (E and F) Adipose depot weights. IWAT, inguinal white adipose tissue. (G-K) Plasma values. (L) Liver TAG. (M) Histologic analysis of liver. Original magnification, $\times 20$; scale bars: $200 \mu \mathrm{m}$. For lipids, the scores were defined as follows: $0:<2 \% ; 1: 2 \%-5 \% ; 2:>5 \%(n=6)$. Data are presented as the mean \pm SEM. ${ }^{*}<<0.05$ compared with vehicle, by $t$ test.

could increase GLP-1 secretion. Two hours after injection of C22:6 NAT or vehicle, FAAH-S268D mice were given a meal of glucose alone or a high-fat meal, and blood was collected 30 minutes later. As expected, C22:6 NAT treatment lowered plasma TAG levels after the high-fat meal (Figure 7F), consistent with impaired lipid absorption (Figure 2C and Figure 6C). Glucose and insulin levels were unaltered with treatment (Figure 7, G and I). Plasma GLP-1 was elevated in mice treated with C22:6 NAT and a high-fat meal, but not with glucose alone (Figure $7 \mathrm{H}$ ), indicating that intestinal lipids were necessary to increase GLP-1 secretion, either because of direct stimulation by the dietary lipid or through costimulation of receptors with $\mathrm{C} 22: 6 \mathrm{NAT}$.

\section{Discussion}

Omega-3 fatty acids are essential for development and can regulate many parameters within the cell, including inflammation and fatty acid oxidation (1), and have been implicated in improving plasma and liver TAG levels $(2,3)$ and decreasing cardiovascular disease risk (5). However, the mechanisms for these benefits are incompletely understood. Here, we show a potentially novel pathway for DHA metabolism, where DHA is conjugated to taurine to form C22:6 NAT, which is then secreted into bile to regulate lipid absorption. C22:6 NAT is an understudied lipid metabolite that may mediate some of the beneficial effects of high DHA intake, such as lowering plasma and liver TAG levels. Using a model of 
A

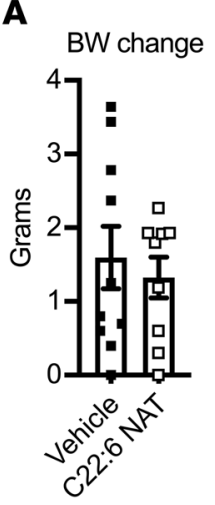

E Liver TAG

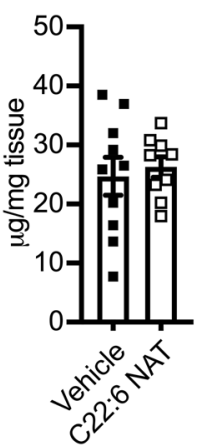

B

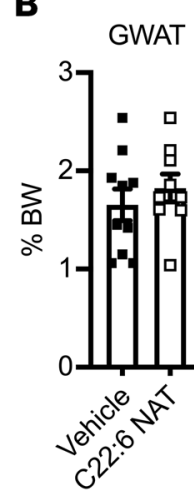

F

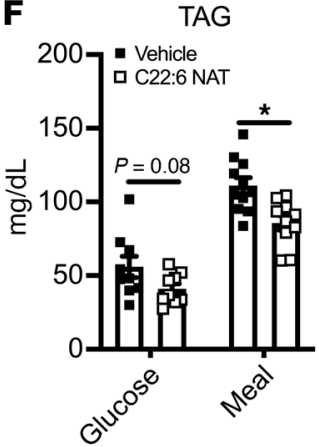

C

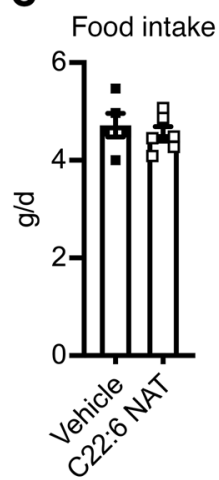

D Plasma

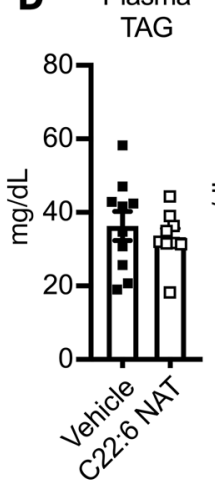

G

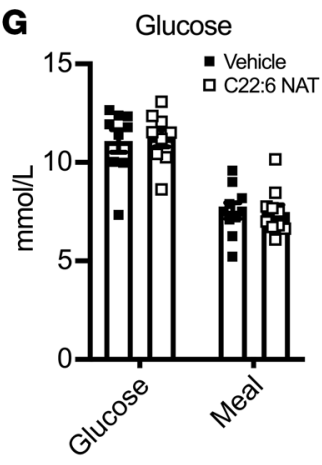

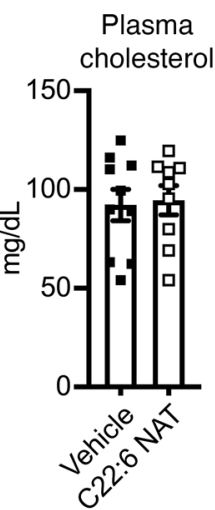
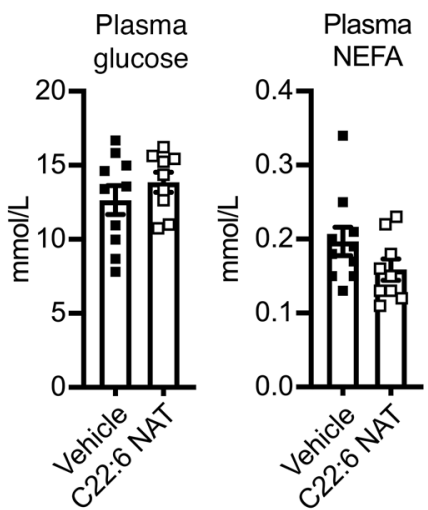

H

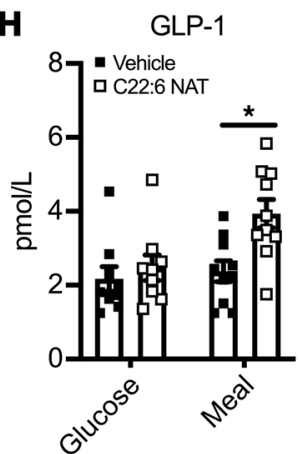

I

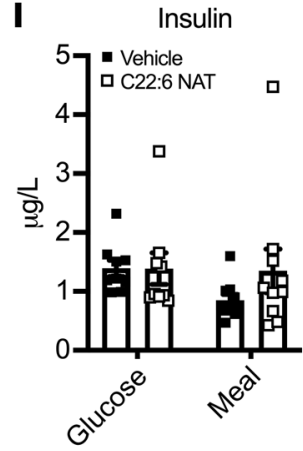

Figure 7. A HSD prevents C22:6 NAT treatment protection against fatty liver. (A-D) FAAH-S268D mice were fed a HSD for 2 weeks and received daily s.c. treatment with C22:6 NAT (10 mg/kg) or vehicle (PBS) $(n=9-10)$. (A) BW change. (B) CWAT weight. (C) Food intake over the last 7 days of the diet ( $n=$ 5-6). (D) Plasma values. (E) Liver TAG levels. (F-I) FAAH-S268D mice fasted for 12 hours were given a s.c. injection of C22:6 NAT (10 mg/kg) or vehicle and, 2 hours later, were dosed orally with glucose or a high-fat mixed meal. Blood was collected 30 minutes after gavage $(n=9-10)$. Data are presented as the mean $\pm \mathrm{SEM} .{ }^{*} P<0.05$ compared with vehicle, by $t$ test.

impaired NAT hydrolysis, we show that treatment with C22:6 NAT itself, and not its components of DHA or taurine, is capable of preventing HFD-induced hepatic lipid accumulation and lowering plasma TAG. Elevated liver and plasma TAG levels are risk factors for cardiometabolic diseases, so understanding the mechanisms behind the existing omega- 3 fatty acid-based treatments can improve future treatments for cardiovascular disease and NAFLD.

NATs are similar to steroidal bile acids in that both can signal through receptors at low concentrations and can be excreted in large quantities into bile to regulate lipid absorption $(9,24)$. The biliary NAT concentrations seen here when mice were given a fish oil-containing diet were in the range of steroidal bile acids, an impressive amount for a class of molecules that has not been previously described in mammalian bile. This high concentration of PUFA-containing NATs could indicate that biliary excretion is a way to minimize oxidative stress in times of excess PUFA consumption or could be a feedback mechanism to limit the amount of lipids that are taken in when the need for essential fatty acids has already been met. Excretion of NATs into bile could be an evolutionarily conserved pathway, as crustaceans, which lack sufficient cholesterol-based bile acids (25), use medium-chain NATs as the primary emulsifier in digestive juice, their equivalent to pancreatic secretions and bile (26). Interestingly, it appears that there is a species difference in the acyl chain, with mammals conjugating taurine to very long-chain fatty acids. This switch likely alters the chemical properties of the NATs, thus probably also changing the functionality of the NATs in the intestine, as evidenced in our study by the actual impairment of lipid absorption in mice with high levels of C22:6 NAT.

We present here a model of moderately impaired lipid absorption, hypometabolism, and normal weight gain but protection from fatty liver development. Separating weight gain and fatty liver is useful for NAFLD research, as low weight gain or weight loss is often a confounding factor in NAFLD development (27). In other models of impaired lipid absorption, such as mice lacking Mogat2 (21) or Cyp8b1 (20), the mice are unable to gain weight normally with HFD feeding, thus preventing conclusions about the direct impact of limited lipid absorption on metabolic parameters. The models of high C22:6 NAT here indicate that impairment of lipid absorption is capable of improving metabolic health, even while gaining weight and adipose mass. This information could indicate that treatment by moderating lipid absorption, without causing weight loss or side effects like steatorrhea, could still have benefits in NAFLD.

Metabolically, mice lacking FAAH are susceptible to HFDinduced hepatic lipid accumulation, insulin resistance, and hypometabolism, and this phenotype has been primarily attributed to the NAEs (14), despite the concurrent high concentration of NATs. Here, we show that FAAH-S268D mice were no more susceptible to weight gain and fatty liver than were FAAH-WT mice 
and were more protected from fatty liver development by fish oil feeding, indicating that NAEs are probably responsible for these detrimental phenotypes seen in the FAAH-KO mice. However, FAAH-S268D mice do show signs of hypometabolism, similar to the FAAH-KO mice (14), which could contribute to weight gain even with lower caloric intake because of impaired lipid absorption. It is currently unclear if this hypometabolism is a consequence of impaired lipid absorption or due to developmental differences. The role of C22:6 NAT in lowering lipid absorption and protecting mice from developing fatty liver is distinct from any known role of other FAAH substrates and is likely due to the chemical properties of C22:6 NAT. As a charged amphipathic molecule, C22:6 NAT can work as a detergent at high concentrations, a function that neutral NAEs are unable to perform, thus allowing us to tease out the functions of NAEs and NATs more clearly.

Interestingly, the acyl chain seems to play a large part in determining a NAT's biological function. C18:1 NAT and C20:4 NAT are unable to lower lipid absorption (Figure 2B), unlike C22:6 NAT. Also, C22:6 NAT does not directly stimulate GLP-1 secretion (Figure 7H), unlike C18:1 NAT (9). Omega-3 fatty acid supplementation also increased C20:5 NAT, albeit to markedly lower levels than C22:6 NAT. This EPA-derived species will be of interest in the future, as EPA and its analogs reduce triglycerides and cardiovascular disease risk (5) and are preferred over DHA supplementation because of potential increased LDL cholesterol with DHA treatment $(28,29)$. Therefore, C20:5 NAT may have different biological effects or mechanisms of action from those of C22:6 NAT, despite both C22:6 NAT and C20:5 NAT increasing with omega-3 fatty acid supplementation. DHA or EPA incorporation into NATs could also affect levels of proresolving mediators such as resolvins or maresin 1, a hydroxylated derivative of DHA, which protects against mice against NASH $(30,31)$. The yet uncertain identification of the in vivo NAT synthase could lead to inhibitor-based approaches to augment the levels of these protective metabolites. These possibilities make the elevation of C20:5 NAT, alone or in combination with C22:6 NAT, an interesting future study to further explore the mechanisms of the beneficial effects of omega-3 fatty acid supplementation.

Because human and murine plasma C22:6 NAT increases with omega-3 fatty acid supplementation, future studies should determine whether DHA supplementation also increases biliary C22:6 NAT in humans, as this could increase our understanding of how omega-3 fatty acids provide benefit. Studies with high omega-3 fatty acid supplementation (approximately $4 \mathrm{~g} /$ day) show that fish oil can inhibit lipid absorption in humans $(32,33)$. Further, pharmacological administration of C22:6 NAT in humans could be a way to slow lipid absorption, which can increase GLP-1 secretion and satiety (34), as well as provide the essential fatty acid DHA and taurine upon hydrolysis.

In conclusion, C22:6 NAT is a DHA-derived metabolite that can limit lipid absorption and prevent hepatic lipid accumulation, providing insight into potentially novel mechanisms for the beneficial effects of omega-3 fatty acids and presenting interesting avenues of treatment for hyperlipidemia and NAFLD.

\section{Methods}

Materials. All chemicals were sourced from Merck/Sigma-Aldrich, unless otherwise specified. Radioactive isotopes were provided by
PerkinElmer. C18:1 NAT was synthesized as previously described (9). C20:4 NAT and C22:6 NAT were synthesized using the same method as for C18:1 NAT, by reacting the appropriate acyl-chloride with taurine (see Supplemental Methods). Both were purified over a silica gel with methanol as an eluent. Fractions containing product were concentrated under reduced pressure. The products' characterization is provided in the Supplemental Methods.

NAT quantification in plasma and bile. Plasma $(150 \mu \mathrm{L})$ was extracted as previously described (9) and injected at $10 \mu \mathrm{L}$ in a random order. Bile $(2.5 \mu \mathrm{L})$ was diluted with $47.5 \mu \mathrm{L} \mathrm{C15:0} \mathrm{NAT} \mathrm{(5} \mathrm{pmol)} \mathrm{in} \mathrm{a} \mathrm{Phenome-}$ nex Verex $9 \mathrm{~mm}$ vial and injected at $2.5 \mu \mathrm{L}$ in a random order. In all cases, NATs were separated using a Waters HSS T3 C18 column $(2.1 \times 100$ $\mathrm{mm}, 1.7 \mu \mathrm{m}$ ) and detected using a Bruker Impact II QTOF operated in full scan ( $m / z: 50-1000)$, in negative ion mode as described previously (9). C18:1 NAT, C20:4 NAT, and C22:6 NAT were quantified using an internal standard-normalized standard curve, with C15:0 NAT serving as the internal standard. In plasma, the standard curve concentrations were as described previously (9). In bile, the standard curve ranged in concentration from $0.025 \mu \mathrm{M}$ to $15 \mu \mathrm{M}$. All other NATs were identified and quantified as previously described (9). With the exception of C20:5 NAT, NATs were detected using XCMS Online (35). C20:5 NAT was detected using Bruker QuantAnalysis software. All NATs were quantified on the basis of C18:1 NAT concentrations.

Animal care. Male C57Bl/6NTac mice were obtained from Taconic at 6 to 8 weeks of age. FAAH-S268D mice on a C57Bl/ 6 background were generated as described previously (9). Mice were housed in a specific pathogen-free environment on a 12-hour light/12-hour dark cycle, and experiments were performed during the light cycle. Mice were maintained on regular chow and had free access to food and water unless otherwise specified. Male mice, between the ages of 7 and 15 weeks were used for experiments, unless otherwise specified. For intestinal permeability studies, mice were gavaged with $600 \mathrm{mg} /$ kg FITC-dextran in water, blood was collected 4 hours later, and plasma fluorescence was measured. For gut transit studies, mice were fasted for 3 hours before gavage with a mixed meal (60\% glucose, $20 \%$ casein, $20 \%$ emulsified soybean oil by kcal) with $10 \%$ charcoal $(w / v)$. Intestinal length and distance to the distal charcoal front were measured 30 minutes after gavage. For complex carbohydrate absorption, mice were fasted for 6 hours starting 2 hours into the light cycle. Mice were given an oral gavage of $2 \mathrm{~g} / \mathrm{kg}$ BW maltodextrin. Blood glucose was measured at the indicated time points using the AlphaTrak 2 glucometer (Zoetis). For fecal lipid studies, mice were fed a HFD (60\% kcal fat; Research Diets, D12492) for 4 weeks. For fish oil diet studies, mice were fed a HFD containing fish oil ( $45 \% \mathrm{kcal}$ from fat, $20 \%$ kcal from fish oil, $0.002 \%$ tert-butylhydroquinone; Research Diets, D18052302) or a matched lard-based diet (Research Diets, D18052303). For carbohydrate-induced fatty liver studies, mice were fed a HSD (60\% kcal from sucrose; Research Diets, D02022703). Body composition was measured by MRI. Indirect gas calorimetry and food intake were measured for individually housed mice in metabolic chambers (TSE Systems). For NAT treatment, C22:6 NAT was solubilized in PBS and injected s.c. at a dose of $10 \mathrm{mg} / \mathrm{kg}$ within 1 hour of the start of the dark cycle. For the C22:6 NAT time course, mice were fasted for 4 hours before injection and tissues collected at the indicated time points. For tissue collection, mice were deeply anesthetized with pentobarbital, exsanguinated into a tube containing EDTA, and tissues were snap-frozen. For diet and treatment studies, mice were 
fasted 4 hours before the collection of blood and tissues. GLP-1 secretion was measured in FAAH-S268D mice fasted for 12 hours, starting 4 hours into the dark cycle. Mice were treated s.c. with C22:6 NAT or vehicle and given a high-fat meal (60\% emulsified soybean oil, $30 \%$ glucose, $10 \%$ casein by kcal) 2 hours later, and blood was collected 30 minutes after the meal. GLP-1 was measured by ELISA (Mercodia).

Lipid absorption. For lipid absorption studies, mice were fasted for 4 hours, starting 2 hours into the light cycle. Mice were lightly anesthetized with isoflurane before i.v. injection of $15 \%$ tyloxapol (3.3 $\mu \mathrm{L} / \mathrm{g}$ ) to prevent lipid uptake into tissues. Blood was collected via tail nick 15 minutes later, and the mice were then given an oral gavage of either olive or sunflower oil (time 0). VLDL secretion was measured in the same way, but without the oil meal, and tyloxapol injection was counted as time 0 . For tracer studies, $\left[{ }^{3} \mathrm{H}\right]$ triolein or $\left[{ }^{14} \mathrm{C}\right]$ oleic acid $(5$ $\mu \mathrm{Ci})$ was added to olive oil along with NAT or vehicle (0.1\% DMSO) as indicated. Blood was collected 1 hour and 2 hours after oil gavage. Intestine was collected after 2 hours and was flushed with $0.5 \mathrm{mM}$ taurocholate before being cut into $2 \mathrm{~cm}$ sections and dissolved in $1 \mathrm{~N}$ sodium hydroxide at $65^{\circ} \mathrm{C}$. Radioactivity was measured using a scintillation counter. For nontracer studies, blood was collected 1, 2, and 4 hours after oil gavage, and TAG was measured in plasma using a colorimetric assay (Randox).

Lipase activity and TAG hydrolysis assays. Lipase activity was determined using purified porcine pancreatic lipase ( $5 \mu \mathrm{g} / \mathrm{reaction})$ in 50 $\mathrm{mM}$ sodium phosphate, $\mathrm{pH}$ 8.0, $5 \mathrm{mM}$ deoxycholate, $10 \%$ isopropanol with $160 \mu \mathrm{M} 4$-nitrophenyl palmitate (4-NPP), and $100 \mu \mathrm{M}$ C18:1 NAT, C20:4 NAT, or C22:6 NAT (36). Reactions were read at $410 \mathrm{~nm}$ every minute for 10 minutes. TAG hydrolysis was determined as described before (37). Briefly, porcine pancreatic lipase was dissolved in $10 \mathrm{mM}$ Tris ( $\mathrm{pH}$ 8.0), $0.15 \mathrm{M} \mathrm{NaCl}$, and $1 \mathrm{mM} \mathrm{CaCl}_{2}$. Colipase was dissolved in $10 \mathrm{mM}$ Tris (pH 8.0) and $0.15 \mathrm{M} \mathrm{NaCl}$. Trace $\left[{ }^{3} \mathrm{H}\right]$ triolein $(7.5 \mu \mathrm{Ci} /$ $\mathrm{mL}$ ) was added to $0.4 \mathrm{mM}$ triolein in assay buffer containing $30 \mathrm{mM}$ Tris ( $\mathrm{pH} 8.0$ ), $1.0 \mathrm{mM} \mathrm{CaCl}_{2}$, and $6 \mathrm{mM}$ taurodeoxycholate and sonicated for 10 minutes. NATs $(100 \mu \mathrm{M})$ were added, and the emulsion was vortexed. Reactions were started by adding $50 \mathrm{ng}$ lipase and $0.5 \mu \mathrm{g}$ colipase and incubated at room temperature for 10 minutes. The reaction was stopped by adding extraction solvent (chloroform/methanol/ heptane, 12.5:14:10, v/v/v) and $50 \mathrm{mM}$ sodium carbonate ( $\mathrm{pH}$ 10.5). A portion of the upper phase was counted in a scintillation counter.

Micelle imaging using cryoelectron microscopy on bovine bile. Dried, unfractionated bovine bile (MilliporeSigma, catalog B3883) was suspended in buffer (30 $\mathrm{mM}$ Tris [pH 8], $1 \mathrm{mM} \mathrm{CaCl}_{2}$ ) at a concentration of $86.96 \mathrm{~g} / \mathrm{L}$. After brief vortexing ( $\sim 3$ seconds, top speed) and centrifugation ( $\geq 20,000 g, 5$ minutes), $25 \mu \mathrm{L}$ supernatant was combined with either $25 \mu \mathrm{L}$ buffer or $25 \mu \mathrm{L}$ buffer containing $25 \mathrm{nmol}$ sodiated C22:6 NAT. The samples were vortexed briefly ( $\sim 3$ seconds, top speed) and then allowed to rest at ambient temperature (approximately $20^{\circ} \mathrm{C}$ ) for more than 1 hour prior to preparation for cryoelectron microscopy (cryo-EM). The sample $(3 \mu \mathrm{L})$ was placed onto a glow discharged grid (Quantifoil Holey Carbon Film, 200 mesh Cu) and prepared for cryoEM using a Vitrobot Mark IV (Thermo Fisher Scientific). Samples were analyzed at magnifications of $\times 29,000$ and $\times 145,000$ using a Tecnai $G^{2} 20$ TWIN (Thermo Fisher Scientific). Micelle size and number were quantified using ImageJ (NIH) (38).

Bile collection. For bile secretion rates, murine bile was collected by cannulating the common bile duct after placing a clamp on the cystic duct to prevent flow of bile from the gall bladder. Bile was collected in preweighed tubes for 20 minutes and stored at $-20^{\circ} \mathrm{C}$ until further analysis. For all other studies, bile was collected from the gall bladder.

Liquid chromatography mass spectrometry bile acid quantitation in bile. Bile diluted 1/400th methanol was diluted to either 1/1200th or $1 / 36,000$ th in a Phenomenex Verex $9 \mathrm{~mm}$ vial containing internal standard solution $(0.3 \mu \mathrm{M}$ of D4 taurolithocholic acid-d4 [TLCA-d4], D4 taurodeoxycholic acid-d4 [TDCA-d4], D6 deoxycholic acid-d6 [DCA-d6], D5 cholic acid-d5 [CA-d5], D4 taurocholic acid-d4 [TCA-d4], D4 tauroursodeoxycholic acid-d4 [TUDCA-d4], D5 lithocholic acid-d5 [LCA-d5], and D4 chenodeoxycholic acid-d4 [CDCA-d4]) in water. Steroidal bile acids were detected and quantified as previously described (39).

Plasma, liver, and fecal lipids. Plasma TAG, glucose, and cholesterol were measured using colorimetric assays (Randox). Plasma ketones were measured using a colorimetric assay (MilliporeSigma). Lipids were extracted from liver or dried feces using a chloroform-methanol extraction (chloroform/methanol/water, 1:1:0.875). Lipids were resolubilized in absolute ethanol and measured using a colorimetric assay (Randox).

Histological analysis. A portion of the left lobe of the liver was fixed in $4 \%$ paraformaldehyde overnight, paraffin-embedded, and sectioned. Sections were then stained with H\&E or Picrosirius red and scored for lipid droplet area, inflammation, necrosis, and fibrosis by trained histologists blinded to the experimental groups. The lipid droplet area was estimated at $\times 10$ magnification in H\&E-stained sections, and the following scoring system was used: $0:<2 \% ; 1: 2 \%-5 \% ; 2:>5 \%$.

Statistics. Data are presented as the mean \pm SEM. Statistical analysis was performed using GraphPad Prism, versions 7 and 8 (GraphPad Software). Significance was determined by 2-tailed Student's $t$ test or 1- or 2-way ANOVA with Bonferroni's multiple-comparison test where appropriate. To compare multiple samples with the control, a multiple-comparison corrected Dunnett's test was used following 1-way ANOVA. Micelle size was compared using a Mann-Whitney $U$ test. A $P$ value of less than 0.05 was considered statistically significant. Significantly different NATs were determined using MetaboAnalyst 4.0 (40). In cases in which 2 conditions were compared, an FDR-corrected $t$ test (FDR $<0.05)$ was used. In the case of comparisons of both diets and genotypes, a 2-way, independent ANOVA corrected with FDR (FDR < 0.05 ) was performed to identify significantly different NATs as a function of the fish oil diet.

Study approval. Human plasma was collected as described with approval of the Suffolk Local Research Ethics Committee (Ipswich, Suffolk, United Kingdom, approval 05/Q0102/181; ref. 17). Written informed consent was received from participants prior to inclusion in the study. All murine protocols were approved by the Danish Animal Experiments Inspectorate and performed according to Animal Research: Reporting of In Vivo Experiments (ARRIVE) standards.

\section{Author contributions}

TJG, SAJT, and MPG conceived and designed the studies. TJG, SAJT, JSS, MVM, TSN, JCBJ, PCC, MEM, BFC, and JTT generated data, key reagents, and samples. TJG, SAJT, and MPG analyzed and interpreted the data. TJG, SAJT, and MPG wrote and revised the manuscript. All authors approved the final version of the manuscript.

\section{Acknowledgments}

The authors acknowledge Lucy Browning, Celia Walker, Susan Jebb, and Annette West for their roles in obtaining plasma 
from human participants given supplemental DHA and EPA. The authors also acknowledge the Core Facility for Integrated Microscopy, Faculty of Health and Medical Sciences, University of Copenhagen for cryo-EM imaging. This study was supported by the Novo Nordisk Foundation Center for Basic Metabolic Research. The Novo Nordisk Foundation Center for Basic Metabolic Research is an independent research center at the University of Copenhagen and is partially funded by an unrestricted donation from the Novo Nordisk Foundation (NNF18CC003490). MEM and MVM were supported by the Mitchell Cancer Institute. BFC was supported by NIH grant DA033760.

Address correspondence to: Matthew P. Gillum, Novo Nordisk Foundation Center for Basic Metabolic Research, University of Copenhagen, Blegdamsvej 3B, DK-2200 Copenhagen, Denmark. Phone: 45.23.64.89.95; Email: gillum@sund.ku.dk.
1. Calder PC. Omega-3 polyunsaturated fatty acids and inflammatory processes: nutrition or pharmacology?. Br J Clin Pharmacol. 2013;75(3):645-662.

2. Yan JH, et al. Omega-3 polyunsaturated fatty acid supplementation and non-alcoholic fatty liver disease: a meta-analysis of randomized controlled trials. Medicine (Baltimore). 2018;97(37):e12271.

3. Musa-Veloso K, et al. Systematic review and meta-analysis of controlled intervention studies on the effectiveness of long-chain omega-3 fatty acids in patients with nonalcoholic fatty liver disease. Nutr Rev. 2018;76(8):581-602.

4. Skulas-Ray AC, et al. Omega-3 fatty acids for the management of hypertriglyceridemia: a science advisory from the american heart association. Circulation. 2019;140(12):E673-E691.

5. Bhatt DL, et al. Cardiovascular risk reduction with icosapent ethyl for hypertriglyceridemia. NEngl JMed. 2019;380(1):11-22.

6. Hoek AM, et al. Icosabutate exerts beneficial effects upon insulin sensitivity, hepatic inflammation, lipotoxicity, and fibrosis in mice. Hepatol Commun. 2019;4(2):193-207.

7. Younossi ZM, et al. Global epidemiology of nonalcoholic fatty liver disease-Meta-analytic assessment of prevalence, incidence, and outcomes. Hepatology. 2016;64(1):73-84.

8. Long JZ, et al. An anatomical and temporal portrait of physiological substrates for fatty acid amide hydrolase. JLipid Res. 2011;52(2):337-344.

9. Grevengoed TJ, et al. $N$-acyl taurines are endogenous lipid messengers that improve glucose homeostasis. Proc Natl Acad Sci. 2019;116(49):24770-24778.

10. Saghatelian A, et al. Assignment of endogenous substrates to enzymes by global metabolite profiling. Biochemistry. 2004;43(45):14332-14339.

11. Tourino C, et al. FAAH deficiency promotes energy storage and enhances the motivation for food. Int J Obes. 2010;34(3):557-568.

12. Lauffer LM, et al. GPR119 is essential for oleoylethanolamide-induced glucagon-like peptide-1 secretion from the intestinal enteroendocrine L-cell. Diabetes. 2009;58(5):1058-1066.

13. Fu J, et al. Oleylethanolamide regulates feeding and body weight through activation of the nuclear receptor PPAR-alpha. Nature. 2003;425(6953):90-93.

14. Brown WH, et al. Fatty acid amide hydrolase abla- tion promotes ectopic lipid storage and insulin resistance due to centrally mediated hypothyroidism. Proc Natl Acad Sci. 2012;109(37):14966-14971.

15. Vaitheesvaran B, et al. Peripheral effects of FAAH deficiency on fuel and energy homeostasis: role of dysregulated lysine acetylation. PLoS One. 2012;7(3):e33717.

16. Holwerda DA, Vonk HJ. Emulsifiers in the intestinal juice of crustacea. Isolation and nature of surface-active substances from Astacus leptodactylus Esch. and Homarus vulgaris L. Comp Biochem Physiol. 1973;45(1):51-58.

17. Browning LM, et al. Incorporation of eicosapentaenoic and docosahexaenoic acids into lipid pools when given as supplements providing doses equivalent to typical intakes of oily fish. Am J Clin Nutr. 2012;96(4):748-758.

18. Padwal RS, Majumdar SR. Drug treatments for obesity: orlistat, sibutramine, and rimonabant. Lancet. 2007;369(9555):71-77.

19. Pignol D, et al. Critical role of micelles in pancreatic lipase activation revealed by small angle neutron scattering. J Biol Chem. 2000;275(6):4220-4224.

20. Higuchi S, et al. Bile acid composition regulates GPR119-dependent intestinal lipid sensing and food intake regulation in mice. Gut. 2020;69(9):1620-1628.

21. Nelson DW, et al. Intestine-specific deletion of acyl-CoA:monoacylglycerol acyltransferase (MGAT) 2 protects mice from diet-induced obesity and glucose intolerance. J Biol Chem. 2014;289(25):17338-17349.

22. Armstrong MJ, et al. Glucagon-like peptide 1 decreases lipotoxicity in non-alcoholic steatohepatitis. J Hepatol. 2016;64(2):399-408.

23. Gastaldelli A, et al. Exenatide improves both hepatic and adipose tissue insulin resistance: a dynamic positron emission tomography study. Hepatology. 2016;64(6):2028-2037.

24. Hofmann AF, Hagey LR. Key discoveries in bile acid chemistry and biology and their clinical applications: history of the last eight decades. J Lipid Res. 2014;55(8):1553-1595.

25. van den Oord A, et al. Absence of bile salts in the gastric juice of a crab. Nature. 1964;(203):301.

26. van den Oord A, et al. On the structure of the emulsifiers in gastric juice from the crab, cancer pagurus L. J Biol Chem.1965;240(5):2242-2247.

27. Friedman SL, et al. Mechanisms of NAFLD devel- opment and therapeutic strategies. Nat Med. 2018;24(7):908-922.

28. Mason RP, et al. Emerging mechanisms of cardiovascular protection for the omega-3 fatty acid eicosapentaenoic acid. Arterioscler Thromb Vasc Biol. 2020;40(5):1135-1147.

29. Chang C, et al. Safety and tolerability of prescription omega-3 fatty acids: a systematic review and meta-analysis of randomized controlled trials. Prostaglandins Leukot Essent Fat Acids. 2018;129:1-12.

30. Han Y, et al. A maresin 1/ROR $\alpha / 12$-lipoxygenase autoregulatory circuit prevents inflammation and progression of nonalcoholic steatohepatitis. JClin Invest. 2019;129(4):1684-1698.

31. Musso G, et al. Bioactive lipid species and metabolic pathways in progression and resolution of nonalcoholic steatohepatitis. Gastroenterology. 2018;155(2):282-302.

32. Harris WS, et al. Reduction of postprandial triglyceridemia in humans by dietary n-3 fatty acids. J Lipid Res. 1988;29(11):1451-1460.

33. Harris WS, Muzio F. Fish oil reduces postprandial triglyceride concentrations without accelerating lipid-emulsion removal rates. Am J Clin Nutr. 1993;58(1):68-74.

34. Cummings DE, Overduin J. Gastrointestinal regulation of food intake. J Clin Invest. 2007;117(1):13-23.

35. Gowda $\mathrm{H}$, et al. Interactive XCMS online: simplifying advanced metabolomic data processing and subsequent statistical analyses. Anal Chem. 2014;86(14):6931-6939.

36. Lewis DR, Liu DJ. Direct measurement of lipase inhibition by orlistat using a dissolution linked in vitro assay. Clin Pharmacol Biopharm. 2012;1:1000103.

37. Lowe ME. Assays for pancreatic triglyceride lipase and colipase. Methods Mol Biol. 1999;109:59-70.

38. Schindelin J, et al. The ImageJ ecosystem: an open platform for biomedical image analysis. $\mathrm{Mol}$ Reprod Dev. 2015;82(7-8):518-529.

39. Morville T, et al. Divergent effects of resistance and endurance exercise on plasma bile acids, FGF19, and FGF21 in humans. JCI Insight. 2018;3(15):e122737.

40. Chong J, et al. Using metaboanalyst 4.0 For comprehensive and integrative metabolomics data analysis. Curr Protoc Bioinforma. 2019;68(1):e86. 